\title{
New Hadroproduction results from the HARP/PS214 experiment at CERN PS
}

\section{M.BONESINI*i}

Sezione INFN Milano Bicocca

E-mail: maurizio.bonesini@mib.infn.it

The HARP experiment at the CERN Proton Synchroton has collected data for hadroproduction measurements with a large set of targets, ranging from hydrogen to lead, in the momentum range 3.0 - $15 \mathrm{GeV} / \mathrm{c}$, covering an extended angular range $(0.025 \leq \theta \leq 2.15 \mathrm{rad})$ with a redundant particle identification. Obtained results may be used for the tuning of neutrino beamline simulations, going from conventional neutrino beams to superbeams and neutrino factories, and for a better understanding of extended air shower and atmospheric neutrinos simulations. In addition, they will be of great interest for the tuning at low energies of the available general-purpose hadronic simulation packages, such as GEANT4, MARS or FLUKA.

European Physical Society Europhysics Conference on High Energy Physics, EPS-HEP 2009, July 16 - 222009

Krakow, Poland

* Speaker.

'on behalf of the HARP Collaboration 


\section{Introduction}

All general-purpose hadronic simulation packages available (GEANT4, MARS, FLUKA) need experimental input for their tuning at low energies, where data are scarce. In addition, the simulation of a conventional $v_{\mu}$ beam with a Monte Carlo (MC) is a delicate task due to complicate cascade processes involved in the neutrino production. The paucity of available hadroproduction data, needed for MC tuning, can limit systematically the precision in the calculations. New hadroproduction data at low energies are also of great interest for extended air showers (EAS) and atmospheric neutrinos simulations and for the neutrino factory (NF) design. One relevant point is how existing MC simulations compare to available hadroproduction data. At low energies $(\leq 15$ $\mathrm{GeV}$ ), the main experimental results come from the HARP experiment [1] at CERN PS and new results will be briefly summarized here. At higher energies we refer to [2] for further details.

\section{The HARP experiment at CERN PS}

The HARP experiment at CERN PS was designed to study hadroproduction on nuclear targets (from $\mathrm{H}_{2}$ to Ta) in the incident momentum range between 3.0 and $15 \mathrm{GeV} / \mathrm{c}$. The HARP detector [3] is shown in Fig. 1 and includes different subdetectors for tracking and particle identification (PID) over the full solid angle. At large angle $\left(20^{\circ} \leq \theta \leq 160^{\circ}\right)$ tracking and PID are performed by a TPC and an array of RPC counters. In the forward direction $\left(\theta \leq 14.3^{0}\right)$ the tracking device is a set of drift chambers, from the previous NOMAD experiment, while the PID is provided by a threshold Cerenkov counter, a large area time of flight wall (TOFW) and an e.m. calorimeter. Beam particles are tagged by a system of beam TOF detectors (TOFA,TOFB) and Cherenkov counters.

Data were taken in 2001 and 2002, for a total of about $420 \times 10^{6}$ triggers in $\sim 300$ experimental settings and are summarized in Fig. 1.

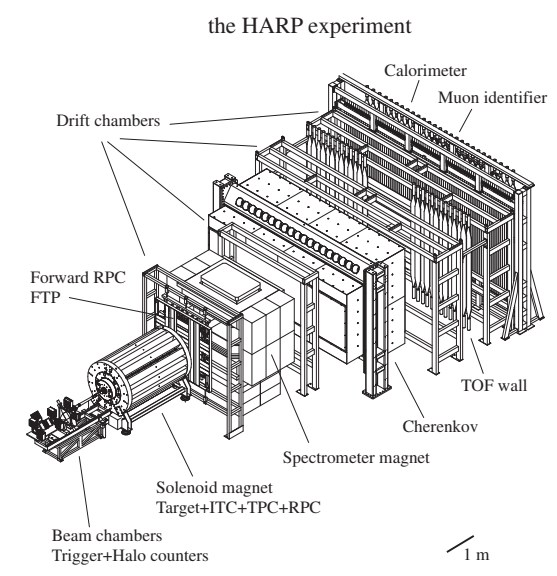

\begin{tabular}{|c|c|c|c|}
\hline Target & $\begin{array}{c}\text { Energy } \\
(\mathrm{GeV})\end{array}$ & $\begin{array}{c}\text { Length } \\
(\lambda)\end{array}$ & $\begin{array}{c}\text { Events } \\
\left(10^{6}\right)\end{array}$ \\
\hline $\mathrm{Be}$ & $3,5,8,12,15$ & $2 \%, 5 \%, 100 \%$ & 37.4 \\
$\mathrm{C}$ & $3,5,8,12,15$ & $2 \%, 5 \%, 100 \%$ & 30.7 \\
$\mathrm{Al}$ & $3,5,8,12,15$ & $2 \%, 5 \%, 100 \%$ & 34.5 \\
$\mathrm{Cu}$ & $3,5,8,12,15$ & $2 \%, 5 \%, 100 \%$ & 36.6 \\
$\mathrm{Sn}$ & $3,5,8,12,15$ & $2 \%, 5 \%$ & 23.7 \\
$\mathrm{Ta}$ & $3,5,8,12,15$ & $2 \%, 5 \%, 100 \%$ & 38.2 \\
$\mathrm{~Pb}$ & $3,5,8,12,15$ & $2 \%, 5 \%, 100 \%$ & 44.9 \\
$\mathrm{~N}$ & $3,5,8,12,15$ & $6 \mathrm{~cm}$ & 13.0 \\
$\mathrm{O}$ & $3,5,8,12,15$ & $6 \mathrm{~cm}$ & 15.5 \\
$\mathrm{H}$ & $3,5,8,12,15$ & $6,18 \mathrm{~cm}$ & 32.0 \\
$\mathrm{D}$ & $3,5,8,12,15$ & $6 \mathrm{~cm}$ & 21.0 \\
$\mathrm{Mini}$ & +8.9 & $5 \%, 50 \%, 100 \%$ & 22.6 \\
$\mathrm{BooNE}$ & & replica & \\
$\mathrm{K} 2 \mathrm{~K}$ & +12.9 & $5 \%, 50 \%, 100 \%$ & 15.3 \\
& & replica & \\
$\mathrm{H}_{2} \mathrm{O}$ & +1.5 & $10 \%, 100 \%$ & 6.4 \\
$\mathrm{~Pb}$ Ta,Cu & +1.5 & $5 \%$ & 3.2 \\
\hline
\end{tabular}

Figure 1: Left panel: layout of the HARP experiment at CERN PS. Right panel: main datasets taken by HARP at CERN PS in 2001-2002. Data were taken at both polarities, except when explicitely stated. 


\section{Large-angle analysis and results for simulation of NF beams.}

The analysis of the large-angle HARP data $(0.35 \leq \theta \leq 2.15 \mathrm{rad})$ is based on the tracks reconstructed by the TPC. They were affected by large distortions mainly in the last part of each spill, due to severe TPC hardware shortfalls that were discovered only after the end of the data taking. After the development of corrections for these effects and the validation of the TPC performance with benchmarks based on real data [4], the entire set of full spill large-angle p-A data has been analyzed and published in reference [5]. Figure 2 shows just an example, where some comparisons with available MC simulations are outlined. None of the considered models describe fully HARP data. However, $\pi^{+}$production is described better than $\pi^{-}$production. At lower (higher) energies binary and Bertini models from GEANT4 (the FTP model from GEANT4 and MARS) seem more appropriate. Parametrized models (such as LHEP from GEANT4) show relevant discrepancies, up to a factor 3. Comparisons with earlier data are available in references [6]. Additional results,

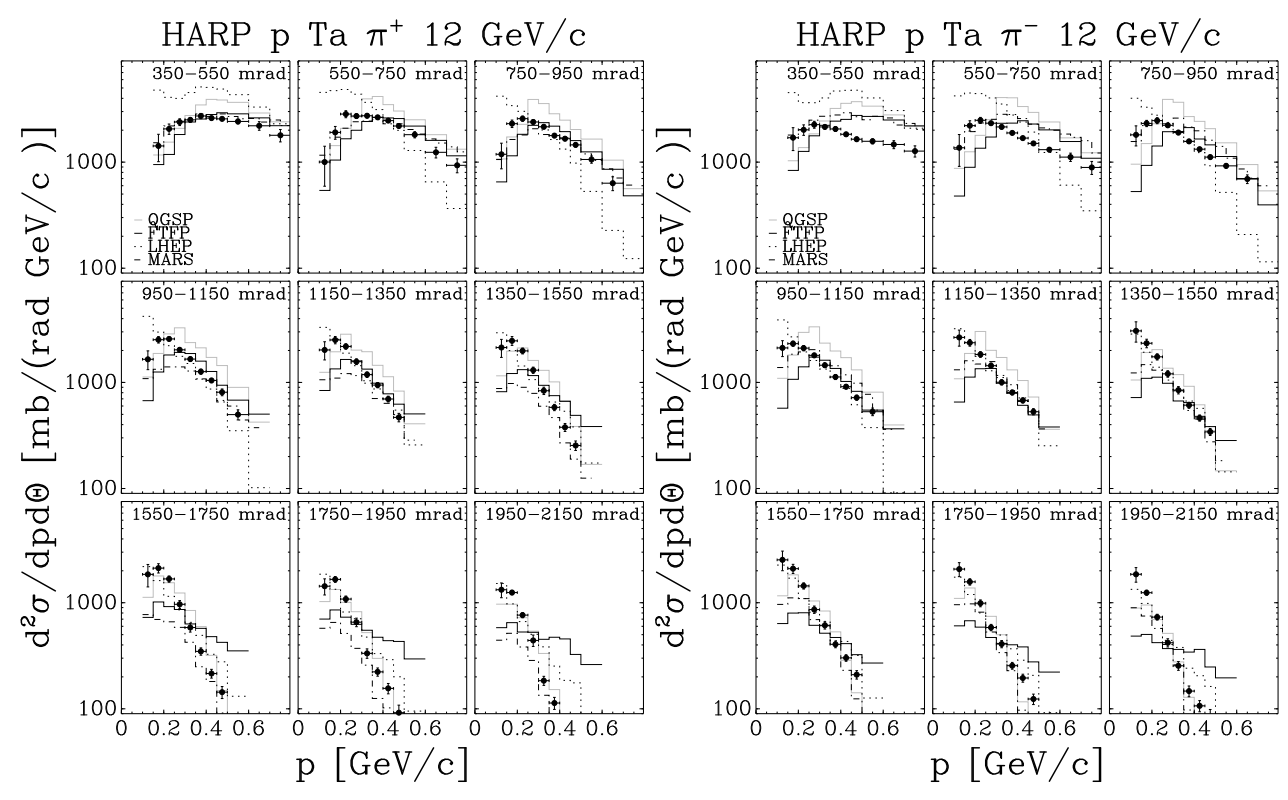

Figure 2: Experimental results from HARP at $12 \mathrm{GeV} / \mathrm{c}$ for $\mathrm{p}$-Ta large-angle cross sections for $\pi^{ \pm}$production, as compared to MC models. See [5] for further details and more data-MC comparisons.

with incoming $\pi^{ \pm}$, are now being obtained in reference [7]. As an example, figure 3 shows the results for $\pi^{+} \mathrm{Ta}$ interactions at $12 \mathrm{GeV} / \mathrm{c}$, as compared to some MC simulations. Additional results to compare the pion yiels with short $\left(5 \% \lambda_{I}\right)$ and long $\left(100 \% \lambda_{I}\right)$ nuclear targets are reported in reference [8].

The baseline option for a NF target is a $\mathrm{Hg}$ jet target with impinging particles at energies $10 \pm 5 \mathrm{GeV}$. Available data are very scarce and for the MC tuning the HARP data on heavy targets, such as $\mathrm{Ta}$ or $\mathrm{Pb}$, are of utmost importance. The kinematical coverage of the HARP experiment is compared with the acceptance of a typical NF design in figure 4 . The experiment covers the full momentum range of interest for production angles bigger than $0.35 \mathrm{rad}$. The pion yield increases linearly with momentum and has an optimum between $4 \mathrm{GeV} / \mathrm{c}$ and $8 \mathrm{GeV} / \mathrm{c}$, as can be seen in the right panel of figure 4. 

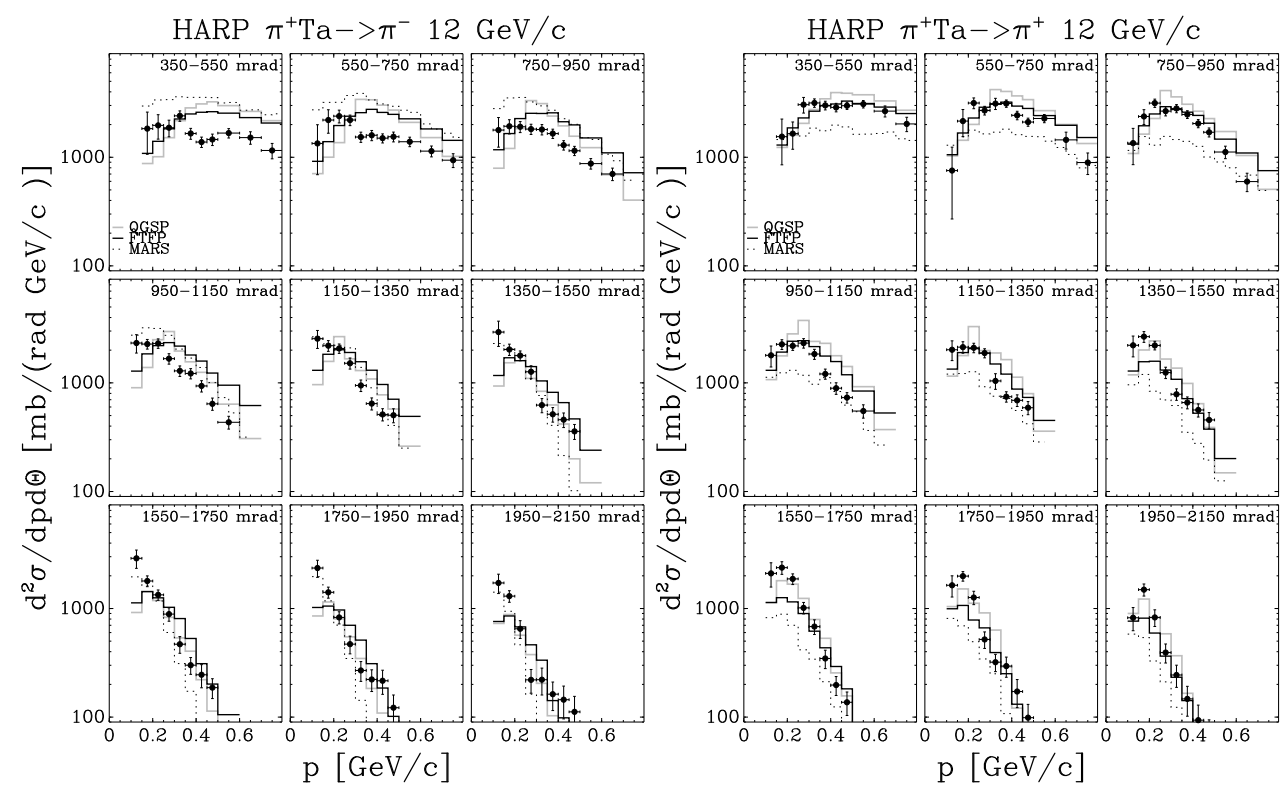

Figure 3: Experimental results from HARP at $12 \mathrm{GeV} / \mathrm{c}$ for $\pi^{+}$-Ta cross sections for $\pi^{ \pm}$production, as compared to MC models.
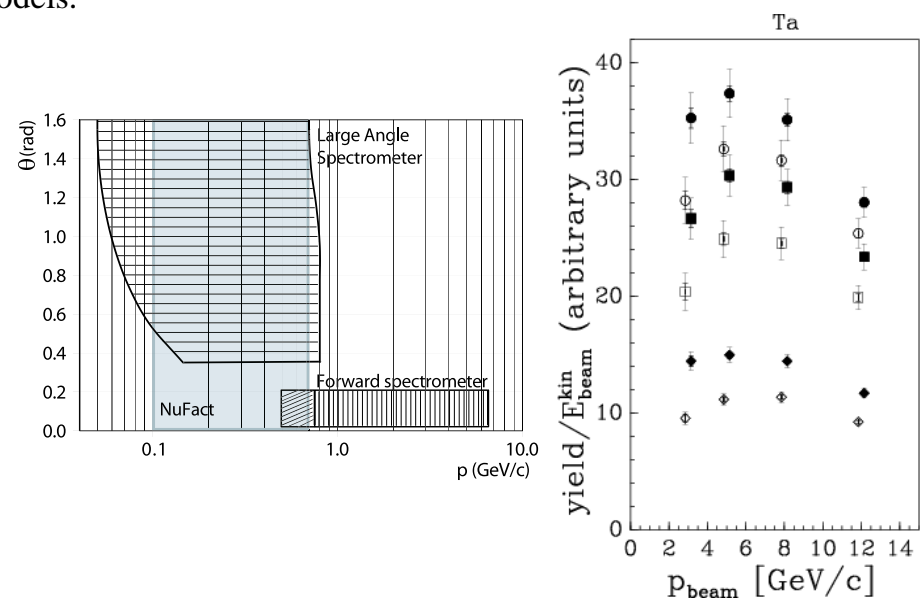

Figure 4: Left panel: kinematic region in the $p-\theta$ plane covered by HARP as compared to the acceptance of the input stage of typical NF designs. Right panel: $\pi^{+}$(closed symbols) and $\pi^{-}$(open symbols) yields: the circles indicate the integral over the full HARP acceptance, the squares are integrated over $0.35 \mathrm{rad}$ $\leq \theta \leq 0.95 \mathrm{rad}$, while the diamonds require in addition the cut $250 \mathrm{MeV} / \mathrm{c} \leq p \leq 500 \mathrm{MeV} / \mathrm{c}$.

\section{Forward analysis: results for the simulation of conventional neutrino beams, atmospheric neutrinos and EAS.}

In the forward region analysis $(\theta \leq 250 \mathrm{mrad})$ tracks are reconstructed in the drift chambers downstream of the magnet, while PID is based on the threshold Cherenkov, the large area timeof-flight TOFW and the calorimeter. The pion identification efficiency is around $98 \%$, while the background from mis-identified protons is well below $1 \%$. On average the total integrated (differential) systematic error is around $5-6(10-11) \%$, with statistical errors of the same order. Final results on p-A and $\pi^{ \pm}$-A interactions have been reported in references [9] and [10]. The full 
set of HARP p-A data (about 1000 exp. points) has been summarized with a Sanford-Wang type parametrization [11], see [9] for details and figure 5 for an example.

Prediction of the far detector spectrum in the absence of oscillations is a key ingredient in a neutrino oscillation experiment. This can be done by an extrapolation from a near detector via a nominal far/near ratio estimated by a beamline MC simulation. The error on the observed number of events in the $\mathrm{K} 2 \mathrm{~K}$ far detector (SuperKamiokande) was dominated by contributions from uncertainties of normalization $( \pm 5 \%)$ and far/near ratio $( \pm 5 \%)$. The previously reported HARP measurements of the $\pi^{+}$production in $\mathrm{p}-\mathrm{Al}$ interactions at $12.9 \mathrm{GeV} / \mathrm{c}$ [12] have contributed in a significant way to reduce the systematic error associated to the FAR/NEAR ratio (from $5.1 \%$ to $2.9 \%$ ), thus increasing the K2K sensitivity to oscillation signals [13]. Similar results were obtained in $8.9 \mathrm{GeV} / \mathrm{c} \mathrm{p}-\mathrm{Be}$ interactions [14] and have contributed to a better understanding of the MiniBooNE and SciBooNE $v$ fluxes. Figure 5 reports the comparison with some available MC models for the $8.9 \mathrm{GeV} / \mathrm{c}$ p-Be data.

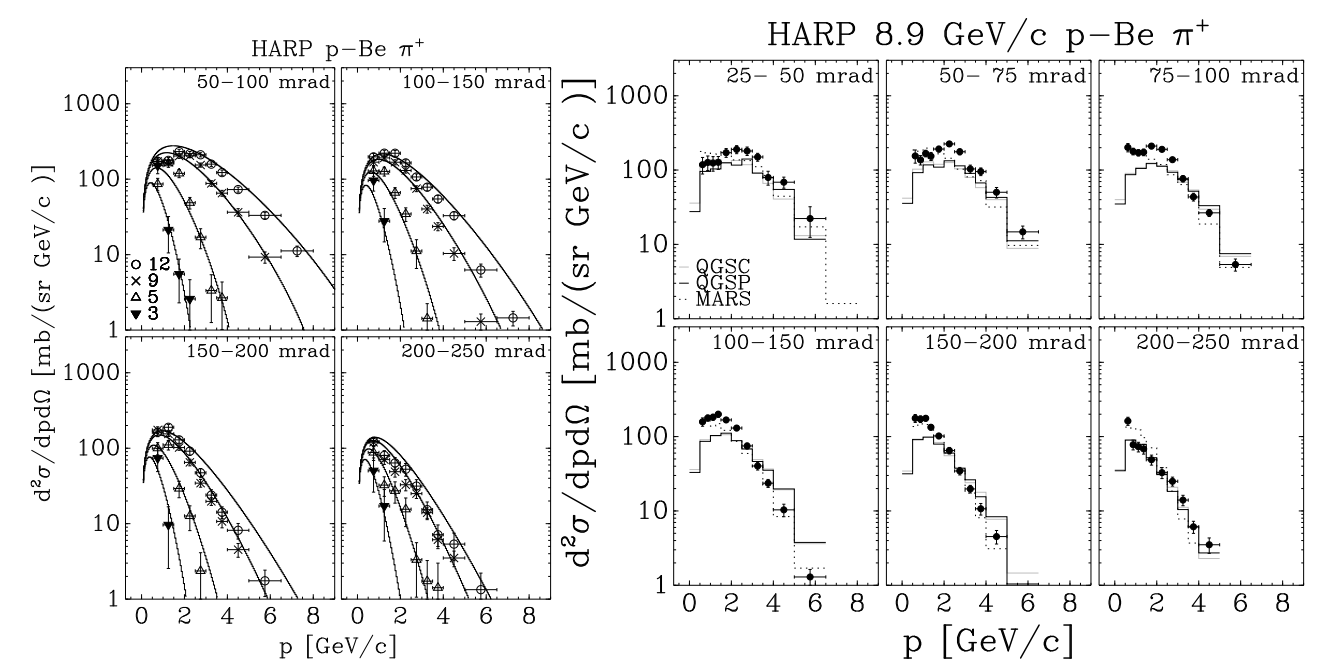

Figure 5: Left: parametrization of p-Be data with a Sanford-Wang parametrization. Right: experimental results from HARP at $8.9 \mathrm{GeV} / \mathrm{c}$ for p-Be cross sections for $\pi^{+}$production, as compared to MC models from GEANT4 (QGSC,QGSP) and MARS.

Results on cryogenic targets, such as $\mathrm{N}_{2}$ and $\mathrm{O}_{2}$ have a direct impact on the precise calculation of atmospheric neutrino fluxes and on the improved reliability of extensive air shower simulations by reducing the uncertainties of hadronic interaction models in the low energy range. In particular, the common hypothesis that $\mathrm{p}-\mathrm{C}$ data can be used to predict the $\mathrm{p}-\mathrm{N}_{2}$ and $\mathrm{p}-\mathrm{O}_{2}$ pion production cross-sections was confirmed. HARP has published results [15] on charged pion production crosssections in interactions of $12 \mathrm{GeV} / \mathrm{c}$ protons on $\mathrm{C}$ and $\mathrm{O}_{2}$ and $\mathrm{N}_{2}$ thin cryogenic targets, in the kinematic range $0.5 \mathrm{GeV} / \mathrm{c} \leq p_{\pi}<8 \mathrm{GeV} / \mathrm{c}$ and $50 \mathrm{mrad} \leq \theta_{\pi}<250 \mathrm{mrad}$. Some results, showing also a comparison with available simulations, are reported in figure 6 .

\section{Conclusions}

The HARP experiment has already made important contributions for a better understanding 

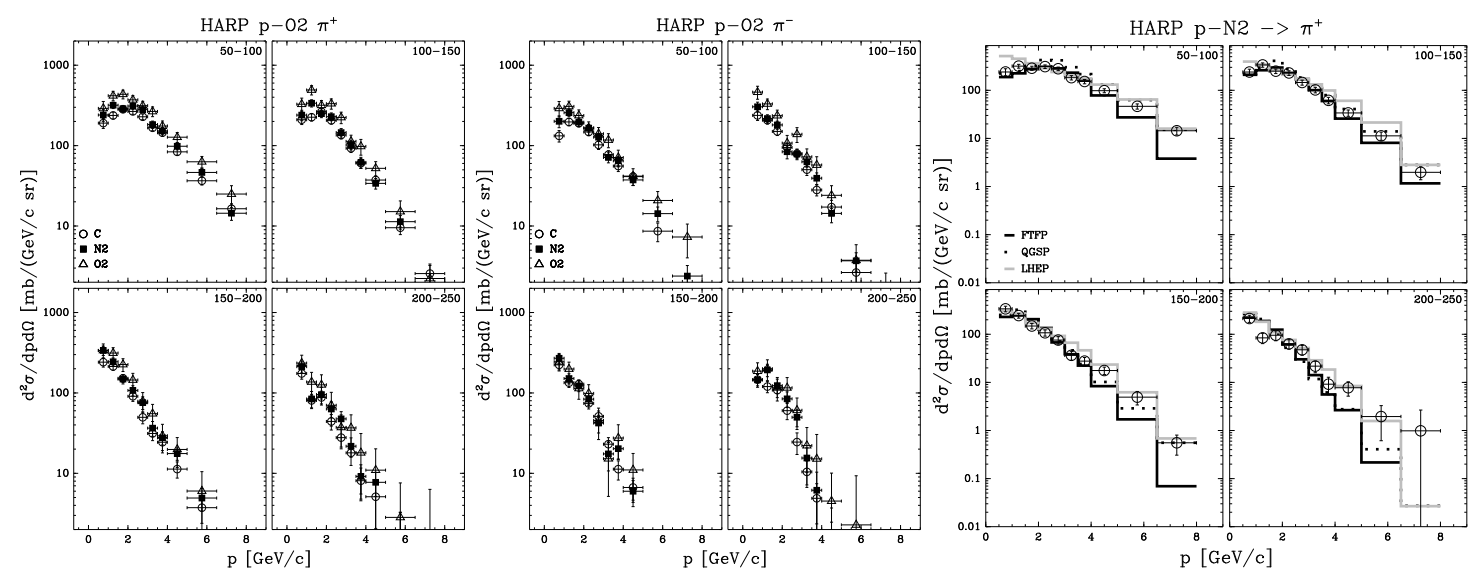

Figure 6: Left: $\mathrm{p}-\mathrm{O}_{2}, \mathrm{p}-\mathrm{N}_{2}, \mathrm{p}-\mathrm{C}$ cross sections at $12 \mathrm{GeV} / \mathrm{c}$; right: comparison of $\pi^{+}$production in $\mathrm{p}-\mathrm{N}_{2}$ interactions with different MC models from GEANT4.

of conventional beamlines, such as $\mathrm{K} 2 \mathrm{~K}$ and MiniBOONE, helping to reduce systematics errors in their oscillation papers, and has given substantial inputs for the neutrino factory project. In addition, results on forward pion production from $\mathrm{N}_{2}, \mathrm{O}_{2}$ targets relevant for atmospheric neutrino simulations and EAS studies were obtained. The extended HARP dataset at low energies is important for the tuning of hadronic interaction packages. New results are ready for publication.

\section{References}

[1] M.G. Catanesi et al., HARP Collaboration, CERN-SPSC/99-35, SPSC/P315, 15 November 1999.

[2] M. Bonesini, A. Guglielmi, Phys. Rep. 433 (2006) 65.

[3] M. G. Catanesi et al., HARP Collaboration, Nucl. Instr. Meth. A571 (2007) 527;

M. Baldo-Ceolin et al., Nucl. Instr. Meth. A532 (2004) 548.

[4] A.Bagulya et al., preprint arXiv:0903.4762 [hep-ex] 2009;

M.G. Catanesi et al., HARP Collaboration, JINST 3 (2008) P04007, arXiv:0907.2806

[5] M.G. Catanesi et al., HARP Collaboration, Phys. ReV. C77 (2008) 0555207

[6] M.G. Catanesi et al., HARP Collaboration, Eur. Phys. J. C51 (2007) 787;

M.G. Catanesi et al., HARP Collaboration, Eur. Phys. J. C53 (2008) 177;

M.G. Catanesi et al., HARP Collaboration, Eur. Phys. J. C54 (2008) 37

[7] M.Apollonio et al., HARP Collaboration, preprint arXiv:0907.1428 [hep-ex] 2009.

[8] M.Apollonio et al., HARP Collaboration, preprint arXiv:0909.8337 [hep-ex] 2009.

[9] M.Apollonio et al., HARP Collaboration, preprint arXiv:0907.3857, to be published on Phys. ReV. C

[10] M.Apollonio et al., HARP Collaboration, Nucl. Phys. A821 (2009) 118.

[11] J.R. Sanford abd C.L. Wang, AGS internal preprint, 1967, unpublished.

[12] M.G. Catanesi et al., HARP Collaboration, Nucl. Phys. B732 (2006) 1.

[13] M.H. Ahn et al., K2K Collaboration, Phys. ReV.D74 (2006) 072003.

[14] M.G. Catanesi et al., HARP Collaboration, Eur. Phys. J. C52 (2007) 49.

[15] M.G. Catanesi et al., HARP Collaboration, Astr. Phys. 29 (2008) 257;

M.G. Catanesi et al., HARP Collaboration, Astr. Phys. 30 (2008) 124. 